\title{
Authors' reply re: Evaluation of treatments for Bartholin's cyst or abscess: A systematic review. (Response to BJOG-20-1118)
}

\author{
James Duffy ${ }^{1}$, Emma Kirk ${ }^{2}$, Benjamin Illingworth ${ }^{3}$, Katie Stocking ${ }^{4}$, and Marian Showell ${ }^{5}$ \\ ${ }^{1} \mathrm{UCL}$ \\ ${ }^{2}$ Royal Free London NHS Foundation Trust \\ ${ }^{3}$ North West Anglia NHS Foundation Trust \\ ${ }^{4}$ University of Manchester \\ ${ }^{5}$ Cochrane Menstrual Disorders and Subfertility Group
}

June 12,2020

Sir,

We would like to thank Sharma and colleagues for their interest in our recent study evaluating the effectiveness and safety of surgical interventions for Bartholin's cyst or abscess. ${ }^{1}$

Their response highlights the unique opportunity offered by randomised trials, and their syntheses into metaanalyses, to assess patient reported outcomes. We would strongly encourage researchers to select, collect and report patient reported outcomes in future research evaluating interventions for Bartholin's cyst or abscess. ${ }^{2}$

The primary outcome should be the outcome of greatest therapeutic importance to the study's prospective hypothesis. There is currently no consensus regarding the selection of outcomes and methods of definition or measurement for randomized trials evaluating interventions for Bartholin's cyst or abscess. ${ }^{3}$ In the absence of a standardized approach, researchers have made arbitrary decisions when choosing among several important outcomes. ${ }^{4}$ It would be useful for healthcare professionals, researchers, and women with lived experience of Bartholin's cyst or abscess to engage in a formal consensus development process to agree appropriate primary and secondary outcomes. ${ }^{3}$

We agree the use of adjuvant antibiotics is an important consideration. They were not reported by any of the included trials. ${ }^{5}$

We have no experience of marsupialization performed under local anaesthetic. In our opinion, this approach would need to be evaluated within a research setting. The recent COVID-19 pandemic would provide additional impetus to undertake this much needed research.

James M. N. Duffy ${ }^{1,2}$, Emma Kirk ${ }^{3}$, BJG Illingworth ${ }^{4}$, K Stocking ${ }^{5}$,

Marian Showell 6

${ }^{1}$ Institute for Women's Health, University College London, London, United Kingdom.

${ }^{2}$ King's Fertility, Fetal Medicine Research Foundation, London, United Kingdom.

${ }^{3}$ Department of Obstetrics and Gynaecology, Royal Free London NHS Trust, London, United Kingdom.

${ }^{4}$ North West Anglia NHS Foundation Trust, Peterborough City Hospital, Peterborough, UK

${ }^{5}$ Centre for Biostatistics, Division of Population Health, Health Services Research and Primary Care, University of Manchester, Manchester, UK 
${ }^{6}$ Cochrane Gynaecology and Fertility Group, University of Auckland, Auckland, New Zealand.

\section{References}

1. Sharma et al. Re: Evaluation of treatments for Bartholin's cyst or abscess: A systematic review. (First comment letter. Reference to be added).

2. Duffy JMN, Bhattacharya S, Herman M, Mol B, Vail A, Wilkinson J, et al. Reducing research waste in benign gynaecology and fertility research. BJOG. 2017;124(3):366-9.

3. Duffy JMN, Rolph R, Gale C, Hirsch M, Khan KS, Ziebland S, et al. Core outcome sets in women's and newborn health: A systematic review. BJOG. 2017 Sep;124(10):1481-9.

4. Duffy JMN, Ziebland S, von Dadelszen P, McManus RJ. Tackling poorly selected, collected, and reported outcomes in obstetrics and gynecology research. Am J Obstet Gynecol. 2019 Jan;220(1):71.e1-.e4.

5. Illingworth B, Stocking K, Showell M, Kirk E, Duffy JMN. Evaluation of treatments for Bartholin's cyst or abscess: A systematic review. BJOG. 2020;127(6):671-8. 